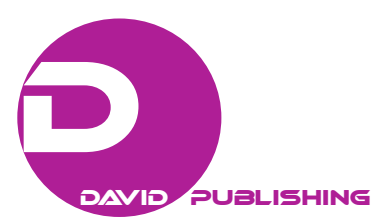

\title{
Toward an Understanding of the Neurofunctional Dissociation between Animal and Tool Concepts: A Graph Theoretical Analysis
}

\author{
Kaoutar Skiker \\ LIST Laboratory, FST, Abdelmalek Essaadi’s University, Tangier, Morocco
}

Mounir Maouene

Department of computer science, ENSAT, Abdelmalek Essaadi’s University, Tangier, Morocco

\begin{abstract}
Neuroimaging studies have shown that animal and tool concepts rely on distinct networks of brain areas. Animal concepts depend predominantly on temporal areas while tool concepts rely on fronto-temporo-parietal areas. However, the origin of this neurofunctional distinction for processing animal and tool concepts remains still unclear. Here, we address this question from a network perspective suggesting that the neural distinction between animals and tools might reflect the differences in their structural semantic networks. We build semantic networks for animal and tool concepts derived from McRae and colleague's behavioral study conducted on a large number of participants. These two networks are thus analyzed through a large number of graph theoretical measures for small-worldness: centrality, clustering coefficient, average shortest path length, as well as resistance to random and targeted attacks. The results indicate that both animal and tool networks have small-world properties. More importantly, the animal network is more vulnerable to targeted attacks compared to the tool network a result that correlates with brain lesions studies.
\end{abstract}

Keywords: animals, tools, network, semantics, small-world, resilience to damage

\section{Introduction}

In neuroscience, brain lesion and neuroimaging studies have shown that the processing of animal and tool concepts depend on distinct brain areas $[1,2,3,4]$. Brain lesions studies report the case of patients with a so-called category-specific deficit, specifically, patients with selective impaired knowledge about animate objects such as animals (four-legged animals) and those with selective impairment for man-made objects such as tools [5]. Category specific deficits for knowledge about animals and tools are attributed to differential

Corresponding author: Kaoutar Skiker, Ph.D. student, Research field: Computational cognitive neuroscience. Informatics, Systems and Telecommunications laboratory (LIST), FST, Abdelmalek Essaadi's University, Tangier 90000, Morocco. Email: skiker.kaoutar85@gmail.com. 
damage to neural systems underlying the representation of perceptual and functional attributes. According to this point of view, deficits in animal category results from lesions in posterior brain areas in temporal and limbic cortex, while deficits in tool category are accompanied with lesions in more anterior brain areas (fronto-parital cortex) $[6,7,8,9]$.

Furthermore, neuroimaging studies on normal, intact individuals have also established that different brain areas are recruited for processing animal and tool concepts [10]. Brain areas that are specifically activated for processing animal stimuli are localized in the temporal cortex, a region known to be implicated in the processing of the visual features of objects [11]. For example, animal stimuli evoke activations in the lateral fusiform gyrus, the medial and inferior occipital regions and the right superior temporal sulcus involved in processing form, color and motion knowledge $[12,13]$. On the other hand, brain areas that are specifically activated for tool stimuli $[14,15,16]$ are localized primarily in anterior motor areas. For example, specific activations for tool stimuli has been found in posterior middle temporal gyrus (pMTG), the medial fusiform gyrus (mFG), the left ventral premotor cortex (PMv) and the left inferior parietal lobe (IPL) [17, 18]. These localizations reflect the fact that identifying tools depend on motor knowledge [19].

The neurofunctional differences between animal and tool concepts have been interpreted in three different manners [20]. First, animal and tool concepts are strongly related to different types of perceptual/functional features [21]. As a consequence, animal concepts rely predominantly on visual features and thus evoke activations in temporal areas involved in processing visual features. In contrast, tool concepts depend strongly on functional features and thus evoke activations in frontal, parietal and temporal areas implicated in processing motor knowledge. Second, there are specialized brain areas for processing animal and tool categories within the ventral visual pathway [22] involved in high order visual processing of objects (e.g., object categorization). These specialized areas might have developed through an evolutionary pressure [23] where similar semantic categories are represented close to each other [24] or emerge from the functional connectivity between these areas and the rest of the brain [25]. For example, pMTG showed connectivity with many of the brain areas shown to be engaged in visual processing of tools and accessing knowledge associated with their use such as mFG, PMv, IPL, etc. Third, animal and tool concepts differ in term of their features correlation and distinctiveness [26]. Animal concepts have more shared features that are highly correlated and thus evoke activation in both posterior and anterior temporal areas for recognizing the domain and the identity of objects, respectively, whereas tool concepts have few shared features and thus activations are detected in posterior temporal area. Taken all together, these three interpretations share the same assumption that animal and tool concepts are processed by a network of different brain areas.

This paper is designed to address the issue of the neurofunctional distinction between animal and tool concepts by making use of graph theory. The way semantic memory is organized, the distances among words and their organization in subcategories remains an open question, and no unified theoretical model is widely 
Concepts: A Graph Theoretical Analysis

accepted [see 27 for an extensive review]. Recently a new model has been proposed in neurocognitive science that uses computational network tools (based on graph theory) founded on the Small World Network Model [28, 29]. In a small-world network the basic components are sub-clusters of nodes and relatively short path length (number of edges connecting two nodes in the network). Networks that have a small world structure always have significantly higher clustering coefficients and smaller average short path lengths than random networks. This type of organization allows for fast search and retrieval of information as well as robustness in the preservation of information and is claimed to capture some of the core properties of networks of meanings [30, 31]. Based on the graph theoretic approach to semantic memory, we propose that the neural distinction between animal and tools might result from the differences in the structural architectures of their respective network. This paper is organized as follow: We first select the animal and tool words as well as their semantic features from McRae and colleague's behavioral study [32], we then build the corresponding semantic networks, with nodes correspond to animal and tool names and edges correspond to a semantic link between nodes (by way of feature overlap). We examine these two networks using of a number of graph theoretical measures including: centrality, clustering coefficient, short path length, small-world topology and resilience to random and targeted attacks. We finally discuss our results in line with previous neuroimaging and brain lesions results.

\section{Data}

Animal and tool words are derived from McRae's database, which consists of a set of 541 living (e.g., animals) and non-living things of different kinds, among which tools, with their corresponding semantic features $(n=2526)$ [32]. These data have been originally obtained from a behavioral study conducted on approximately 725 participants, with 30 participants providing features for each concept. The participants in that study were given each noun and 14 blank spaces to fill with features and were prompted to provide physical properties (how it looks, smells, sounds, etc.), functional properties or uses, internal properties, and other pertinent facts. The networks of animal and tool words are then build, with nodes representing animals vs tool words and edges connecting these nodes if a they share at least one common semantic feature (eg. 'cow' and 'horse' are linked to each other because they share at least one feature 'has_legs').

\section{Method}

Networks (graphs) $\mathrm{G}=(\mathrm{V}, \mathrm{E})$ are a mathematical tool, with nodes corresponding to entities, in our case words used to speak about animals and tools, and edges corresponding to relationships between them, in our case semantic links that exist between two words. Graphs can be directed/undirected and weighted/unweighted. In this work, both animal and tool networks are undirected and unweighted. 


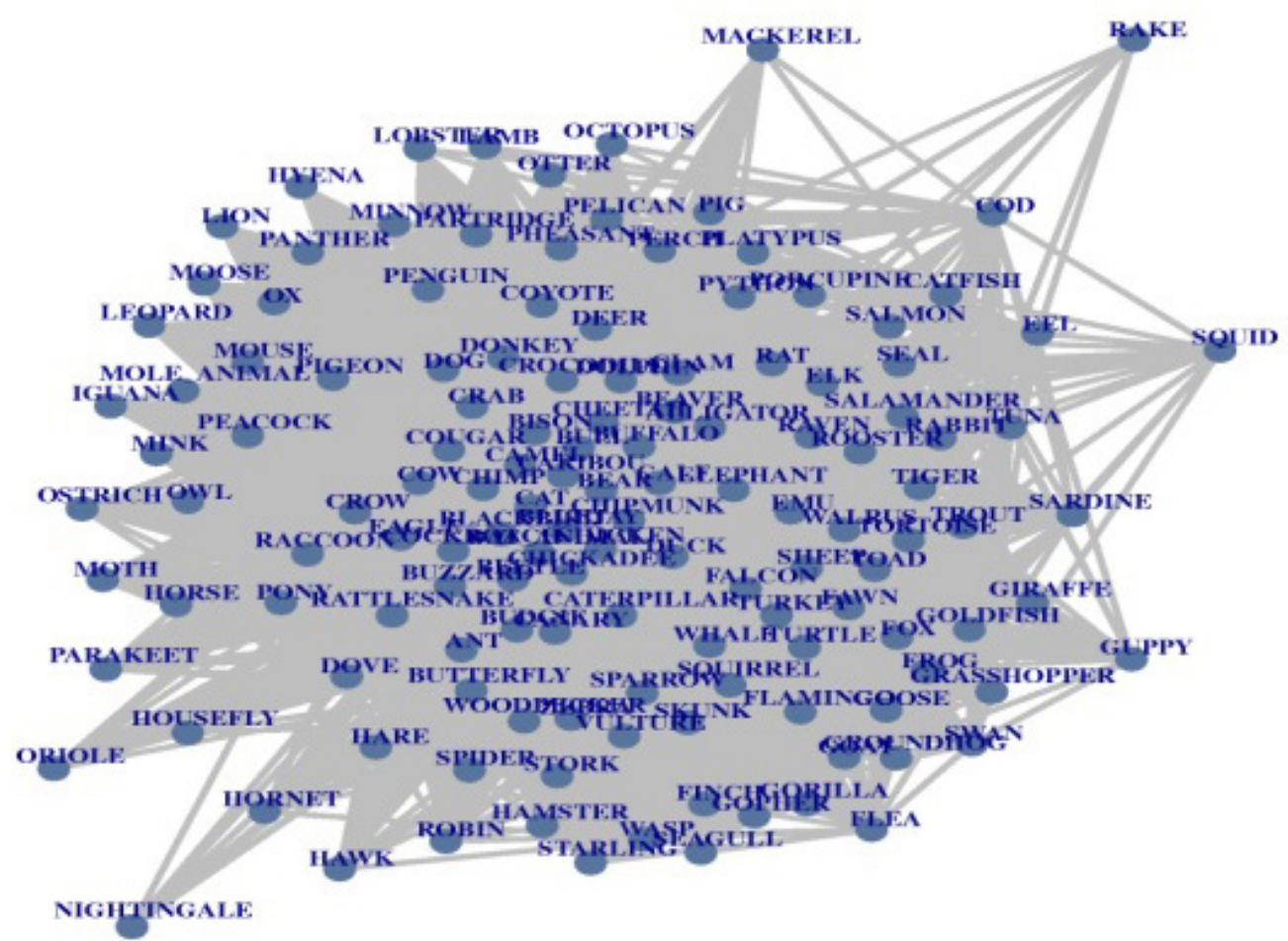

Figure 1. Animal network composed of 137 words and 7662 semantic links.

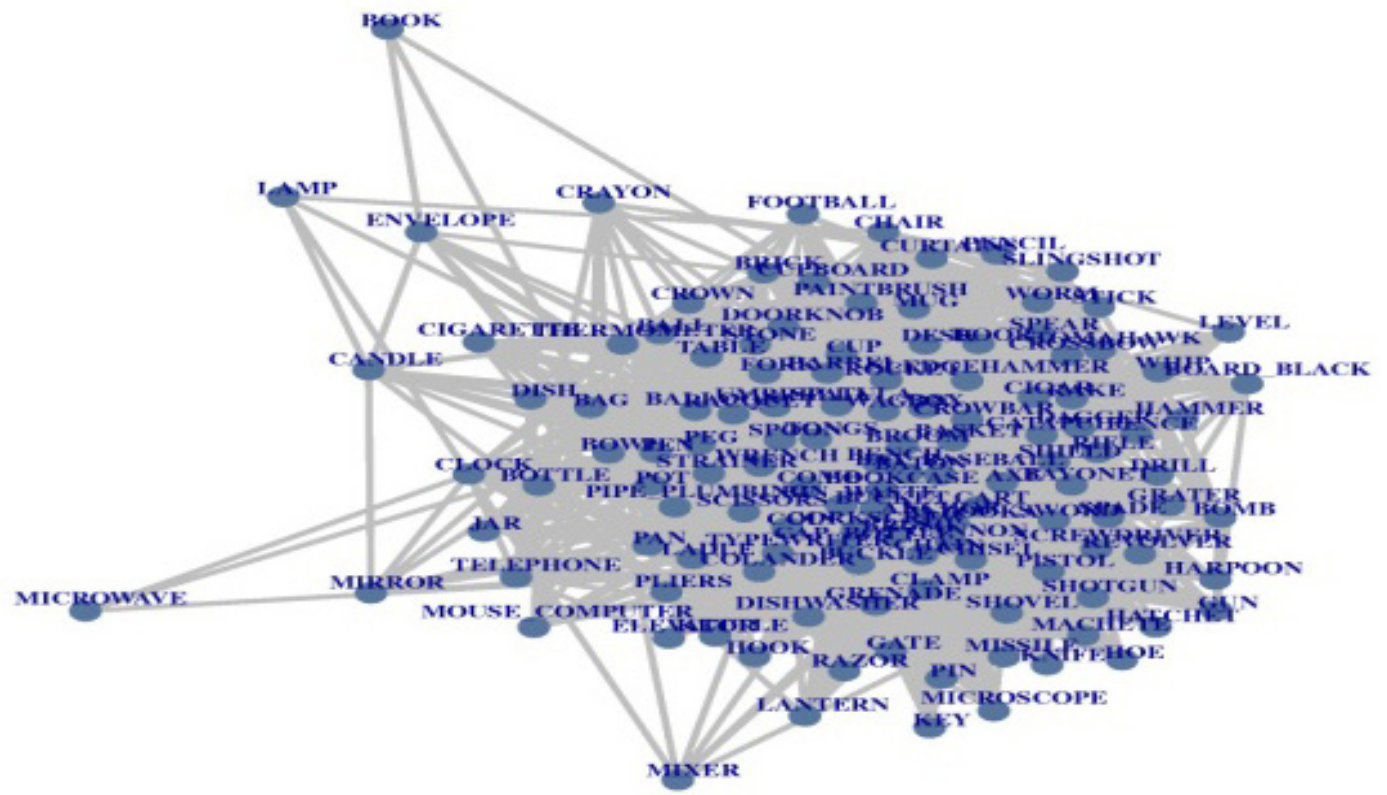

Figure 2. Tool network composed of 127 words and 4924 semantic links.

\section{Topological Measurments}

The networks of animals and tools are assessed with regard to a number of graph measures such as centrality, clustering coefficient, efficiency, assortativity, path length. Such graph measures allow for the characterization of the topological architecture of these two networks by identifying the contribution of 
Toward an Understanding of the Neurofunctional Dissociation between Animal and Tool Concepts: A Graph Theoretical Analysis

individual nodes; for example the existence of high degree nodes, known as hubs, that permits the assessment of the global efficiency of the whole network. This in turn can indicate the capacity of the subnetwork to integrate information in an efficient manner; these measures also consider the small worldness and thus the resilience to damage of such structures [33]. Here, we provide some definition of graph measures used in our study.

(1) Degree centrality: The degree k of each node indicates the number of connections linked to it. Nodes with many edges, or high degree, are considered as hubs which are known to play an important role in the system's dynamics. The Degree distribution shows the fraction of vertices in the network that have degree $\mathrm{k}$ and can provide key information about which vertices are more central than others and are susceptive to more damage if attacked.

(2) Global efficiency captures the number of connections that must be traversed to connect any two nodes.

(3) Clustering coefficient (C) is a measurement of the efficiency of local connectivity.

(4) Characteristic path length (L) is the average minimum number of connections edges that must be traversed to go from one node to another.

(5) Small world analysis [29]: Small-world properties of the networks are examined according to the C and L measured in the above steps. The network exhibits a small world property if the clustering coefficient (C) is greater that the clustering coefficient of a random network $(\mathrm{Cr})$ with the same number of nodes and edges and the characteristic path length (L) is the same as the path length of a random network (Lr). Typically, a small world network must have the following conditions [34]:

$$
\gamma>>1, \lambda \approx 1 \text { and } \sigma>1
$$

All the computations were performed in MatLab Graph theory measures using the brain connectivity toolbox freely available at (https://sites.google.com/site/bctnet/).

Table 1

Overview of the measurements and their signification used in this study

\begin{tabular}{ll}
\hline character & signification \\
\hline $\mathrm{C}$ & clustering coefficient which measures the extent of a local cluster of the network $(0<\mathrm{C}<1)$ \\
$\mathrm{L}$ & path length which measures of the extent of average connectivity of the network \\
$\gamma$ & the ratio of the clustering coefficients between real and random network \\
$\lambda$ & the ratio of the path length between real and random network \\
$\sigma$ & scalar quantitative measurement of the small-worldness of a network \\
$\mathrm{E}$ & a measure of the global efficiency of parallel information transfer in the network \\
$\mathrm{r}$ & a measure of the correlation between the degree of a node and the mean degree of its nearest neighbors \\
\hline
\end{tabular}

(6) Resilience to random and targeted attacks: A second goal of the study is to evaluate the resilience of a network to failures (the random removal of nodes or edges) and attacks (the removal of nodes and edges with high importance). For example, we studied the effect of random failure of nodes or targeted attack of highly 
central hubs on both network structure and dynamics. To this end, we use Nexcade, a freely available program for analysis a various perturbations on networks [35]. We proceed as follows: Words having the highest degree were eliminated and the associated changes on network topology were evaluated. Then the next highest degree nodes were identified and eliminated and the changes in the network topology were recorded. This process was repeated until all nodes of the network had been removed.

(7) The assortativity coefficient is another used measure of network resilience. It quantifies the correlation between the degrees of connected nodes. Assortative networks with positive $\mathrm{r}$ and closer to 1 indicate the existence or a resilient core of interconnected high degree hubs. In contrast, dissortative networks with negative $r$ and closer to -1 indicate the existence of more vulnerable high degree hubs.

\section{Results}

\section{Non randomness, Centrality}

Table1 shows that animal and tool networks differ along a number of dimensions. Figures 3 and 4 show that both animal and tool networks have a non-Gaussian degree distribution indicating the non-randomness of these two networks.

We analyze the characteristics of the degree of centrality in the animal and tool networks and compare them with the corresponding random networks. The aim is to determine the central animals and tool words in the animal and tool networks. "Penguin" was found to be the most central word in the animal network and "spatula"\& "umbrella" were the central most words in the tool network.

\section{Clustering coefficient}

The clustering coefficient of tool network $(C=0.794043)$ is low compared to that of the animal network $(C=0.887424)$ which indicates high local efficiency of information transfer and robustness in the animal network.

\section{Small-World analyses}

Both animal and tool networks have a small-world property as indicated in Table 2. However, the small-worldness of animal network is greater than that of the tool network, as indicated by the scalar $\sigma$, which is interpreted as higher cliqueness (more substructures) and more rapid information transfer within the network of animal compared to the tool network. 
Toward an Understanding of the Neurofunctional Dissociation between Animal and Tool Concepts: A Graph Theoretical Analysis

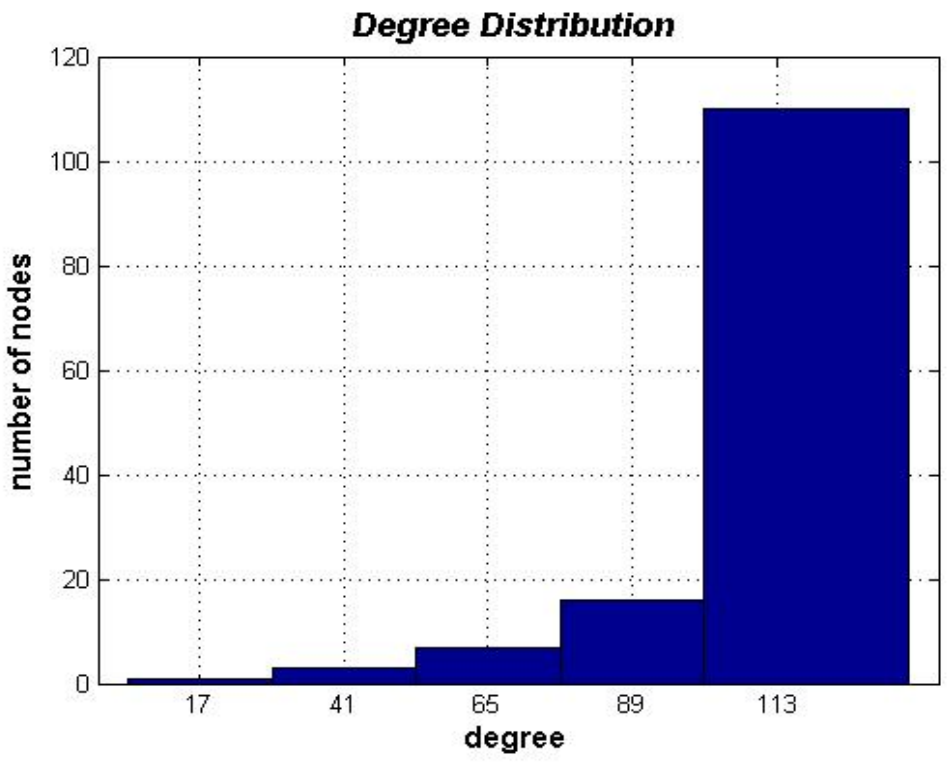

Figure 3. The degree distribution in animal network.

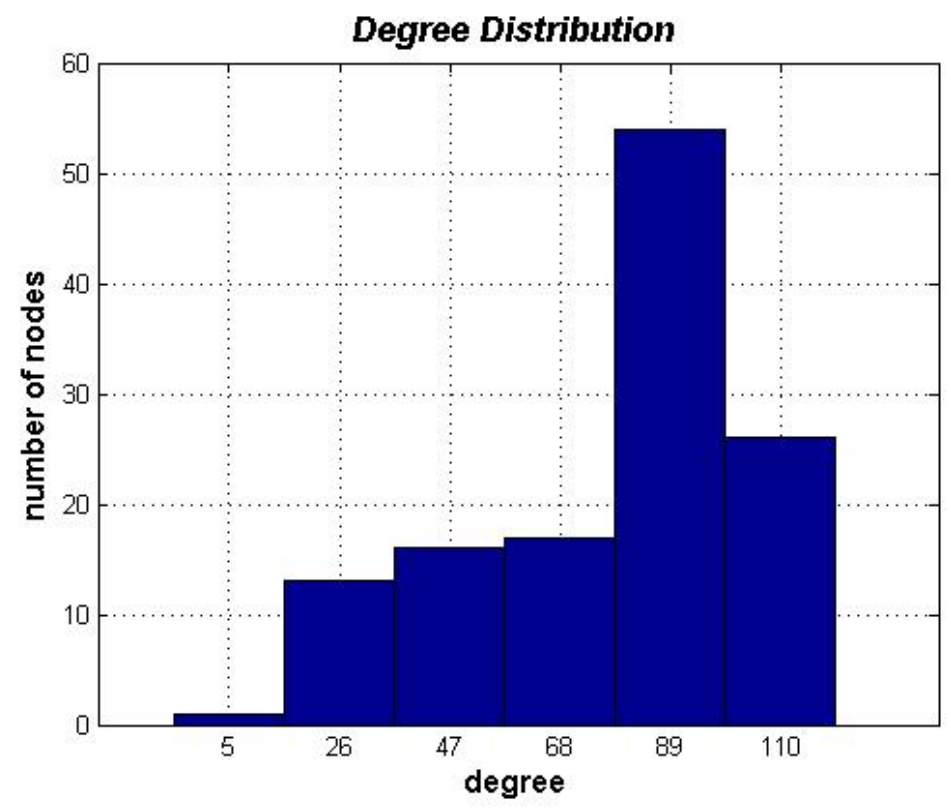

Figure 4. The degree distribution in tool network.

Table2

The small world property of animal and tool networks

\begin{tabular}{llllll}
\hline & $\mathrm{C}$ & $\gamma=\mathrm{C} / \mathrm{Cr}$ & $\mathrm{L}$ & $\lambda=\mathrm{L} / \mathrm{Lr}$ & $\sigma=\gamma / \lambda$ \\
\hline Animal network & $\mathrm{C}=0.8874$ & \multirow{2}{*}{1.7255} & $\mathrm{~L}=1.1691$ & 0.7868 & 2.1931 \\
Random network & $\mathrm{Cr}=0.5143$ & & $\mathrm{Lr}=1.4859$ & & 1.6731 \\
Tool network & 0.7940 & 1.5481 & 1.3752 & 0.9253 & 1.4863 \\
Random network & 0.5129 & & & \\
\hline
\end{tabular}


Table3

Graph theoretical measures

\begin{tabular}{lllll}
\hline & Average degree $<\mathrm{k}>$ & Average $\mathrm{CC}<\mathrm{C}>$ & Efficiency(E) & Assortativity(r) \\
\hline Animal network & 111.854 & 0.8874 & 0,91 & $-0,003$ \\
Tool network & 77.5433 & 0.7940 & 0.80 & 0,091 \\
\hline
\end{tabular}

\section{Resilience to failure and attacks}

The results indicate that both animal and tool networks are robust to random failures but highly sensitive to failures in nodes of high centrality.

Next, words having the highest degree were eliminated and the associated changes on network topology were evaluated. Then the next highest degree nodes were identified and eliminated and the changes in the network topology were recorded. This process was repeated until all nodes of the network had been removed.

We have demonstrated that tool networks are more resistant to attacks that animal network which correlates with brain lesions studies showing that deficits occur more often for animals than tools.

\section{Conclusions}

In this paper, we addressed the issue of why animal and tool concepts rely on a network of distinct brain areas, with animal concepts depending on temporal areas known to contain memory traces of object's visual features and tool concepts depending on fronto-temporo-parietal areas that encompass the representation of motor knowledge. We suggest that this neurofunctional dissociation might result from the differences between the structural architectures of networks representing the semantic relations among animal and tool concepts.

Our analyses show that both animal and tool networks have small world properties indicating the balance between local clustering and rapid information transfer compared to random networks with the same number of nodes and edges.

We have examined the characteristics of the degree of centrality in the two networks and compare them with corresponding random networks. The main characteristic of centrality measures is to determine the more central nodes in the networks. It was found that "Penguin" is the most central word in the animal network, whereas "Umbrella" and "Spatula" are more prominent in the tool network. This means that these words play a prominent role in the transfer of information within the networks. Also, the animal network contains a greater number of nodes with high degree compared to the tool network. In the animal network, there are approximately 100 animal words ranked as the most central ones, all of which have a degree equal to 113 . In the tool network, there are approximately 50 tool words, all of which have the degree equal to 89 . However, the connectivity between high degree nodes is stronger in tool network compared to animal network, as indicated by the asssortativity measure. This means that animal networks are more vulnerable to damage than tool network. To further investigate the robustness of the networks, we examine the effect of the removal of nodes with high degree on the network's dynamic. Our results indicate that animal networks are more vulnerable to 
targeted attacks than tool networks. These results correlate with brain lesions studies showing that deficits in animals occur more often than tools $[6,7,8,9,36]$.

In this study, we have addressed the issue of the neurofunctional dissociation between animal and tool concepts by making use of a graph theory. We have shown that the semantic network of animals differ from those of tools with high small-worldness and high global efficiency within the first and more resilience to damage for the second.

\section{References}

G. Gainotti, "What the locus of brain lesion tells us about the nature of the cognitive defect underlying category-specific disorders: a review", Cortex, vol. 36, no 4, p. 539-559, sept. 2000.

A. Martin, "The representation of object concepts in the brain", Annu Rev Psychol, vol. 58, p. 25-45, 2007.

C. Gerlach, “A review of functional imaging studies on category specificity”, J Cogn Neurosci, vol. 19, nº 2, p. 296-314, févr. 2007.

R. F. Goldberg, C. A. Perfetti, et W. Schneider, "Distinct and common cortical activations for multimodal semantic categories", Cogn Affect Behav Neurosci, vol. 6, n” 3, p. 214-222, sept. 2006.

C. Blundo, M. Ricci, et L. Miller, "Category-specific knowledge deficit for animals in a patient with herpes simplex encephalitis", Cogn Neuropsychol, vol. 23, nº 8, p. 1248-1268, déc. 2006.

S. M. Brambati, D. Myers, A. Wilson, K. P. Rankin, S. C. Allison, H. J. Rosen, B. L. Miller, et M. L. Gorno-Tempini, “The anatomy of category-specific object naming in neurodegenerative diseases", J Cogn Neurosci, vol. 18, n 10, p. 1644-1653, oct. 2006.

M. Laine, J. O. Rinne, J. Hiltunen, V. Kaasinen, et H. Sipilä, "Different brain activation patterns during production of animals versus artefacts: a PET activation study on category-specific processing”, Brain Res Cogn Brain Res, vol. 13, n 1, p. 95-99, févr. 2002.

T. Okada, S. Tanaka, T. Nakai, S. Nishizawa, T. Inui, N. Sadato, Y. Yonekura, et J. Konishi, "Naming of animals and tools: a functional magnetic resonance imaging study of categorical differences in the human brain areas commonly used for naming visually presented objects", Neurosci. Lett., vol. 296, nº 1, p. 33-36, déc. 2000.

D. Perani, S. F. Cappa, V. Bettinardi, S. Bressi, M. Gorno-Tempini, M. Matarrese, et F. Fazio, "Different neural systems for the recognition of animals and man-made tools", Neuroreport, vol. 6, n 12, p. 1637-1641, août 1995.

P. Vitali, J. Abutalebi, M. Tettamanti, J. Rowe, P. Scifo, F. Fazio, S. F. Cappa, et D. Perani, “Generating animal and tool names: an fMRI study of effective connectivity”, Brain Lang, vol. 93, n 1, p. 32-45, avr. 2005.

A. C. Connolly, J. S. Guntupalli, J. Gors, M. Hanke, Y. O. Halchenko, Y.-C. Wu, H. Abdi, et J. V. Haxby, "The representation of biological classes in the human brain”, J. Neurosci., vol. 32, nº 8, p. 2608-2618, févr. 2012.

R. F. Goldberg, C. A. Perfetti, et W. Schneider, "Perceptual Knowledge Retrieval Activates Sensory Brain Regions", J. Neurosci., vol. 26, nº 18, p. 4917-4921, mars 2006.

I. Bramão, L. Faísca, C. Forkstam, A. Reis, et K. M. Petersson, "Cortical brain regions associated with color processing: an FMRI study", Open Neuroimag J, vol. 4, p. 164-173, 2010.

L. L. Chao et A. Martin, "Cortical regions associated with perceiving, naming, and knowing about colors", J Cogn Neurosci, vol. 11, no 1, p. 25-35, janv. 1999.

L. L. Chao et A. Martin, "Representation of manipulable man-made objects in the dorsal stream", Neuroimage, vol. 12, $\mathrm{n}^{\circ} 4, \mathrm{p}$. 478-484, oct. 2000.

S. H. Creem-Regehr et J. N. Lee, “Neural representations of graspable objects: are tools special?”, Brain Res Cogn Brain Res, vol. $22, n^{\circ} 3$, p. 457-469, mars 2005.

S.-A. Rueschemeyer, D. van Rooij, O. Lindemann, R. M. Willems, et H. Bekkering, "The function of words: distinct neural correlates for words denoting differently manipulable objects", J Cogn Neurosci, vol. 22, nº 8, p. 1844-1851, août 2010.

C. Gerlach, I. Law, et O. B. Paulson, "When action turns into words. Activation of motor-based knowledge during categorization of manipulable objects", J Cogn Neurosci, vol. 14, nº 8, p. 1230-1239, nov. 2002. 
B. Z. Mahon, S. C. Milleville, G. A. L. Negri, R. I. Rumiati, A. Caramazza, et A. Martin, "Action-related properties of objects shape object representations in the ventral stream", Neuron, vol. 55, nº 3, p. 507-520, août 2007.

B. Z. Mahon et A. Caramazza, "What drives the organization of object knowledge in the brain? The distributed domain-specific hypothesis", Trends Cogn Sci, vol. 15, no 3, p. 97-103, mars 2011.

M. L. Kellenbach, M. Brett, et K. Patterson, “Actions speak louder than functions: the importance of manipulability and action in tool representation”, J Cogn Neurosci, vol. 15, n 1, p. 30-46, janv. 2003.

L. L. Chao, J. V. Haxby, et A. Martin, "Attribute-based neural substrates in temporal cortex for perceiving and knowing about objects", Nat. Neurosci., vol. 2, n 10, p. 913-919, oct. 1999.

B. Z. Mahon et A. Caramazza, "What drives the organization of object knowledge in the brain? The distributed domain-specific hypothesis", Trends Cogn Sci, vol. 15, nº 3, p. 97-103, mars 2011.

A. G. Huth, S. Nishimoto, A. T. Vu, et J. L. Gallant, "A Continuous Semantic Space Describes the Representation of Thousands of Object and Action Categories across the Human Brain”, Neuron, vol. 76, nº 6, p. 1210-1224, déc. 2012.

R. M. Hutchison, J. C. Culham, S. Everling, J. R. Flanagan, et J. P. Gallivan, "Distinct and distributed functional connectivity patterns across cortex reflect the domain-specific constraints of object, face, scene, body, and tool category-selective modules in the ventral visual pathway", Neuroimage, vol. 96, p. 216-236, août 2014.

L. K. Tyler, S. Chiu, J. Zhuang, B. Randall, B. J. Devereux, P. Wright, A. Clarke, et K. I. Taylor, “Objects and categories: feature statistics and object processing in the ventral stream", J Cogn Neurosci, vol. 25, n 10, p. 1723-1735, oct. 2013.

S. L. Rogers et R. B. Friedman, “The Underlying Mechanisms of Semantic Memory Loss in Alzheimer's Disease and Semantic Dementia », Neuropsychologia, vol. 46, no 1, p. 12-21, janv. 2008.

S.Milgram, “The Small-World Problem”,Psychology Today,vol.1, no 1 ,p.61-67,May 1967.

D. J. Watts et S. H. Strogatz, « Collective dynamics of 'small-world' networks”, Nature, vol. 393, no 6684, p. 440-442, juin 1998.

M. Steyvers et J. B. Tenenbaum, "The large-scale structure of semantic networks: statistical analyses and a model for semantic growth ", oct. 2001

J. Borge-Holthoefer et A. Arenas, "Semantic Networks: Structure and Dynamics”, Entropy, vol. 12, no 5, p. 1264-1302, mai 2010.

K. McRae, G. S. Cree, M. S. Seidenberg, et C. Mcnorgan, "Semantic feature production norms for a large set of living and nonliving things", Behavior Research Methods, vol. 37, n 4, p. 547-559, nov. 2005.

M. E. J. Newman, "The structure and function of complex networks", SIAM REVIEW, vol. 45, p. 167-256, 2003.

M. D. Humphries et K. Gurney, “ Network 'Small-World-Ness': A Quantitative Method for Determining Canonical Network Equivalence ", PLoS ONE, vol. 3, n 4, p. e0002051, avr. 2008.

G. Yadav et S. Babu, "NEXCADE: Perturbation Analysis for Complex Networks", PLoS ONE, vol. 7, n 8, p. e41827, août 2012.J Cogn Neurosci, vol. 25, n 10, p. 1723-1735, oct. 2013.

A. S. Chan, D. P. Salmon, et J. De La Pena, "Abnormal semantic network for "animals" but not "tools" in patients with Alzheimer's disease", Cortex, vol. 37, no 2, p. 197-217, avr. 2001. 